\title{
Detection of Subtype Blood Cells using Deep Learning
}

Tiwari, Prayag; Qian, Jia; Li, Qiuchi; Wang, Benyou; Gupta, Deepak; Khanna, Ashish; Rodrigues, Joel J.P.C.; de Álbuquerque, Victor Hugo C.

\section{Published in:}

Cognitive Systems Research

Link to article, DOI:

10.1016/j.cogsys.2018.08.022

Publication date:

2018

Document Version

Peer reviewed version

Link back to DTU Orbit

Citation (APA):

Tiwari, P., Qian, J., Li, Q., Wang, B., Gupta, D., Khanna, A., Rodrigues, J. J. P. C., \& de Albuquerque, V. H. C. (2018). Detection of Subtype Blood Cells using Deep Learning. Cognitive Systems Research, 52, 1036-1044. https://doi.org/10.1016/j.cogsys.2018.08.022

\section{General rights}

Copyright and moral rights for the publications made accessible in the public portal are retained by the authors and/or other copyright owners and it is a condition of accessing publications that users recognise and abide by the legal requirements associated with these rights.

- Users may download and print one copy of any publication from the public portal for the purpose of private study or research.

- You may not further distribute the material or use it for any profit-making activity or commercial gain

- You may freely distribute the URL identifying the publication in the public portal 


\section{Accepted Manuscript}

Detection of Subtype Blood Cells using Deep Learning

Prayag Tiwari, Jia Qian, Qiuchi Li, Benyou Wang, Deepak Gupta, Ashish Khanna, Joel J.P.C. Rodrigues

PII:

S1389-0417(18)30376-0

DOI: $\quad$ https://doi.org/10.1016/j.cogsys.2018.08.022

Reference: $\quad$ COGSYS 708

To appear in: $\quad$ Cognitive Systems Research

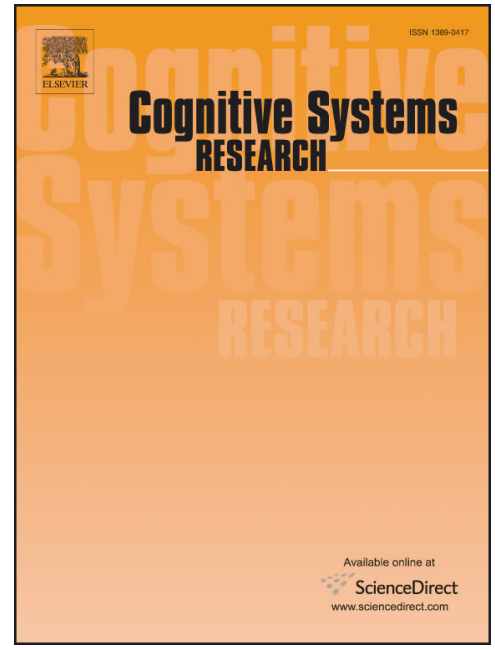

Revised Date: $\quad 13$ August 2018

Accepted Date: $\quad 25$ August 2018

Please cite this article as: Tiwari, P., Qian, J., Li, Q., Wang, B., Gupta, D., Khanna, A., Rodrigues, J.J.P., Detection of Subtype Blood Cells using Deep Learning, Cognitive Systems Research (2018), doi: https://doi.org/10.1016/ j.cogsys.2018.08.022

This is a PDF file of an unedited manuscript that has been accepted for publication. As a service to our customers we are providing this early version of the manuscript. The manuscript will undergo copyediting, typesetting, and review of the resulting proof before it is published in its final form. Please note that during the production process errors may be discovered which could affect the content, and all legal disclaimers that apply to the journal pertain. 


\title{
Detection of Subtype Blood Cells using Deep Learning
}

\author{
Prayag Tiwari ${ }^{\mathrm{a}}$, Jia Qian ${ }^{\mathrm{b}}$, Qiuchi $\mathrm{Li}^{\mathrm{a}}$, Benyou Wang ${ }^{\mathrm{a}}$, Deepak Gupta ${ }^{\mathrm{c}}$, \\ Ashish Khanna $^{\mathrm{c}}$, Joel J. P. C. Rodrigues ${ }^{\mathrm{d}, 1, *}$ \\ ${ }^{a}$ Department of Information Engineering, University of Padova, Italy \\ ${ }^{b}$ Department of Applied Mathematics and Computer Science, Denmark Technology \\ University, Denmark \\ ${ }^{c}$ Maharaja Agrasen Institute of Technology, Delhi \\ ${ }^{d}$ National Institute of Telecommunications, (Inatel), Santa Rita do Sapucai-MG, Brazil \\ Instituto de Telecomunicações, Portugal, ITMO University, St. Petersburg, Russia
}

\begin{abstract}
Deep Learning has already shown power in many application fields, and is accepted by more and more people as a better approach than the traditional machine learning models. In particular, the implementation of deep learning algorithms, especially Convolutional Neural Networks (CNN), brings huge benefits to the medical field, where a huge number of images are to be processed and analyzed. This paper aims to develop a deep learning model to address the blood cell classification problem, which is one of the most challenging problems in blood diagnosis. A CNN-based framework is built to automatically classify the blood cell images into subtypes of the cells. Experiments are conducted on a dataset of $13 \mathrm{k}$ images of blood cells with their subtypes, and the results show that our proposed model provide better results in terms of evaluation parameters
\end{abstract}

Keywords: Blood Cells, Classification, CNN

\footnotetext{
${ }^{2}$ Fully documented templates are available in the elsarticle package on CTAN

* Corresponding Author.

Email addresses: prayag.tiwari@unipd.it (Prayag Tiwari), lainey.qian@gmail.com (Jia Qian), qiuchi.li@studenti.unipd.it (Qiuchi Li), wabyking@163.com (Benyou Wang), deepakgupta@mait.ac.in (Deepak Gupta), ashishkhanna@mait.ac.in (Ashish Khanna), joeljr@ieee.org (Joel J. P. C. Rodrigues)
} 


\section{Introduction}

The white blood cell (WBC), also called leukocytes, is a cellular component of the blood with a nucleus and without a hemoglobin. As an essential part of the immune system, it moves from blood to tissue and provide defense 5 for fighting against the invasion of the foreign microorganisms, e.g., bacteria, viruses, and germs, by ingesting them, destroying infectious agents or by producing antibodies. The leukocyte can be categorized into five genres: Eosinophils, lymphocytes, Neutrophils, Monocytes and Basophils. The Neutrophils are the most abundant, and they are responsible for defending the bacteria or fungal infection. Eosinophils occupy around $2 \%-4 \%$ of WBC, and act in response to allergies and parasite infection. Lymphocytes undertakes the task of the specific recognition of foreign agents and the consequent removal from the host. Monocytes are effective in direct destruction of pathogens and cleanup of the debris from the infection sites. The counter of different white blood cells plays a significant role in the clinical diagnosis and test: it is an indicator that reflects the hidden infection within the body and alerts the hematologists as a signal, i.e., the abnormal increase in WBC is the so-called leukocytosis. It also helps doctors monitor the effectiveness of chemotherapy or radiation treatment in people with cancer $[1]$.

The detection and distinguishment of diverse WBC and the further counting of the corresponding proportion is critical due to the richness of clinical meaning behind it. It is painstaking and of low-efficiency if we manually differentiate the leukocytes under the microscopes, from which the automatic classification based on the images of WBC emerges [2]. Usually, the automatic classification approaches are present with several main steps: preprocessing, segmentation, feature extraction and classification. The preprocessing procedure primarily refers to the attempt of removing the noises or some artifacts from the images to output the contrast images. The segmentation can be considered as the operation of segmenting the WBC from the background of the smear images or extracting the region of interest (ROI). The consequent step is to build a 
representative feature vector for every type of WBC, and the classification will work based on it. In this very step, the hematologist sometimes may be involved to determine the features.

In this paper, we propose a CNN-based model which gives 94 percent accuracy for the 2 labels i.e. polynuclear and mononuclear, 78 percent accuracy for the 4 labels i.e. eosinphil,lymphocyte, neutrophil and monocyte. our model can automatically classify the blood cell type in order to save time and enhance clinical efficiency. The rest of the paper is organized as follows: Section 1 describes the introduction, section 2 describes about the used baseline models to compare with our proposed model, Section 3 provides the literature survey where past relevant work is introduced, Section 4 provides the proposed methodologies followed with architecture and algorithm, Section 5 provides the data records, Section 6 provides the experiment followed by used setups and result analysis with the discussion, and Section 6 provides the conclusion and possible future works.

\section{Background}

In this section, some details about Naive Bayes and SVM has been described because these methods are used as a baseline approach to compare with our proposed model.

\subsection{Naive Bayes}

Bayes theorem with the independence supposition between the predictors is the base of the Naive Bayes classifier. Bayes theorem presents us a way to compute the posterior probability, $P(y \mid x)$ from the $P(y), P(x)$ and $P(x \mid y)$.

Naive Bayes classifier infer that there is in-dependency among each predictor for the given class. This assumption is often known as conditional in-dependency of class and given equation provide better understanding,

$$
P(y \mid x)=\frac{P(x \mid y) P(y)}{P(x)}
$$


where, posterior probability of target for the given attribute or predictor is represented by $P(y \mid x)$, prior probability of the target is represented by $P(y)$, likelihood that the probability of attribute for given class is represented by $P(x \mid y)$ and prior probability of attribute or predictor is represented by $P(X)$.

\subsection{Support Vector Machine}

SVM is used for classification as well as for regression purpose also. The

60 main aim of SVM for classification purpose is to build a hyperplane in a multi dimensional space that partition the cases of distinct class labels. For building a optimal hyperplane, SVM utilize a iterative algorithm during training session, which is utilized to diminish the error function. SVM is classified into 2 types with respect to the type of error function.

SVM 1: Minimization of error function persist during the training session and can be written as:

$$
\frac{v^{T} v}{2}+\text { Const } \sum_{k=1}^{M} \theta_{k}
$$

which subjects to the limitations:

$$
y_{k}\left(v^{T} \varphi\left(x_{k}\right)+b\right) \geq 1-\theta_{k}, \theta_{k} \geq 0, k=1,2, \ldots \ldots, M
$$

${ }_{65}$ Where Const means capacity constant, $b$ is constant as well, $v$ is the vector coefficients, $\theta_{k}$ represents the parameter for supervising the non-separable input data, $y \in\{+1,-1\}$ describes the labels of the class, independent variable is represented by $x_{k}$. The kernel $\varphi$ transform from the input data to the feature space. Larger the value of $C$ so the error can be penalized more that is why $c$ must be opted carefully which could be helpful to avoid over-fitting.

SVM 2: In contrast to the SVM 1, SVM 2 helps to diminish the error function:

$$
\frac{v^{T} v}{2}-w \rho+\frac{1}{M} \sum_{k=1}^{M} \theta_{k}
$$

which subjects to the limitations:

$$
y_{k}\left(v^{T} \varphi\left(x_{k}\right)+b\right) \geq \rho-\theta_{k}, \theta_{k} \geq 0, k=1,2, \ldots \ldots, M, \text { and }, \rho \geq 0
$$




\section{Literature Survey}

The identification of WBC has been studied and explored by many researchers and practitioners with the implementation of different methodologies. Image processing techniques are largely involved in this procedure before applying classification algorithms, such as separating the region of interest from the background, locating the nucleus of WBC (called nucleoli) and even in the preprocessing, removing and cleaning the noises from the microscopic images. To some extent, the segmentation from the background is critical as claimed by [3], and many approaches have come up to address it, such as clustering so ([4]), thresholding ([5, 6, 7]), morphological operator ([8, 9]), Gram-Schmidt orthogonalization method ([10]), edge detection ([11), region growing ([12]), watershed ([13]), colors ([14], [15], [16]) and so on.

A review based on the segmentation techniques ([17]) argues that the conventional color-based and the thresholding methods are simple at the sacrifice of the accuracy, whereas the methods like region growing may offer a high precision with a high-computation cost. Referring to the color-based segmentation methods, some methods work directly on the RGB color space, whilst others instead on HSI or CMYK color space. In general, the S-component-based methods outperform the RGB-based ones. [18] attempts to build the feature vector by leveraging the CMYK color models. They find out that all the other components except white blood cells have some amount of yellow color present in them, whereas leukocytes show a good contrastion in the Y component of CMYK color model.

Our method will bypass the stringent demand of constructing a good feature profile by studying and analyzing the pixels of the microscopic images directly. As the procedure of hand-engineered features could be tricky but also critical, the expertise in different domains is required. However, sometimes the suggestion from the domain-based specialists may also have their own biases or subjective preferences. Our method takes the pixels of images (of three channels, RGB) as the input, and an algorithm automatically learns and harvests 
the critical variables during the training. The variables may be morphological or related to the color, but the algorithm will take good care of them no matter what. This preferable attribute differentiates our method from the work mentioned above by the other researchers.

\section{PRINCIPLE OF CONVOLUTIONAL NEURAL NETWORKS}

\subsection{Methodology}

CNN is a category of Artificial Neural Network (ANN), which has been proven to be useful in image classification and object recognition. It takes the raw pixels as input and produces an outcome indicating the probabilities that the input belongs to different classes. CNN gains its high reputation by advocating an innovative architecture that may address a big hurdle existing in the conventional neural network: a massive amount of parameters to tune in the training stage. Instead of implementing the fully-connected structure in every layer, CNN imposes two additional layers, convolution and pooling, that may significantly reduce the magnitude of parameters. Convolution layer is entitled by the convolutional operation, with the purpose of extracting the features from the input images. Besides, the kernel (filter) size and stride need to be determined in advance. The kernel is a numeric matrix, on which an element-wise operation between it and the input image is carried out. In the example in Figure 1, the green matrix represents the pixel of the original image, the yellow matrix is the kernel or filter, whereas the pink matrix is the convolved feature matrix. The pink matrix is computed by moving the red dash square across the green matrix with the pre-set stride. The modification of kernel generates the features that have the variant effects, e.g., identification of the object, the edge detection, sharpen the image, etc. The pooling procedure is also called sub-sampling or down-sampling, which is designed to reduce the convolved features produced from the convolution operator with the incentive of remaining the considerable information. There are different pooling techniques, for instance, maximum, average, summation, etc. We use the maximize pooling 
in our method, an example is illustrated in Figure2. In a fully-connected layer, one node is connected to all the nodes in the previous layer. Besides, more than one hidden layer may apply, and the output layer may use the diverse classifying operator, e.g., softmax, rectified linear unit, etc.
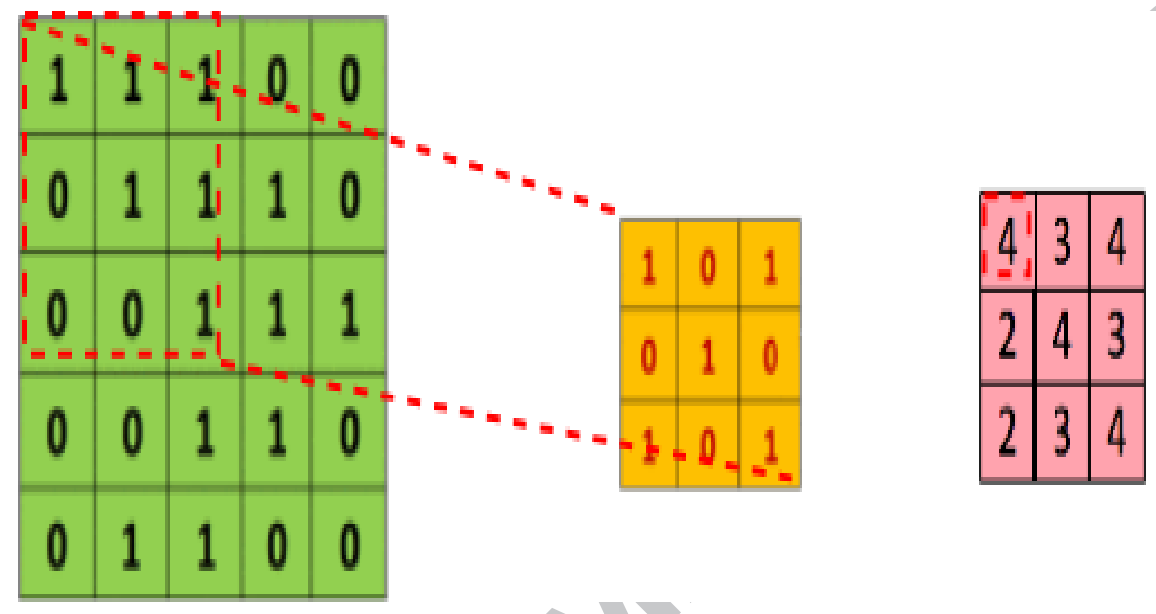

Figure 1: An example of convolution operation: image pixel matrix, kernel and convolved feature (source)

\subsection{Proposed Architecture}

The architecture of our proposed model is described in figure 3 It contains two convolutional layers and one pooling layer, and it is followed by the fullyconnected structure with one hidden layer and output layer. All the detailed settings are listed as the follows:

1. Two convolution layers

- First layer: Kernel size: 3x3, number of output filters: 32, initializer: Glorot uniform

- Second layer: Kernel size: 3x3, number of output filters: 64 , initializer: Glorot uniform

- Activation function: ReLu

- Stride: 1 


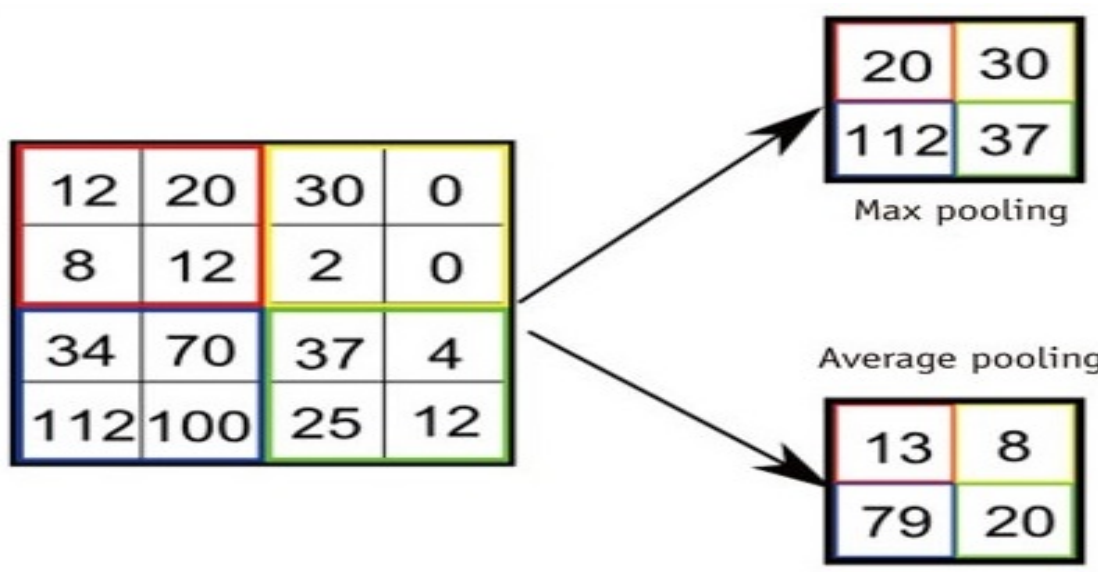

Figure 2: An example of max pooling and average pooling operation (source)

- Input image size: 60x80 (3 channels)

2. Pooling Layer

- Pooling type: Maximize

- Pool size: 2x2

- Dropout: 0.25

3. Fully-connected Layer: Hidden Layer

- Number of nodes:

- Activation Function: ReLu

4. Fully-connected Layer: Output Layer

- Number of nodes: number of classes

- Activation Function: Softmax

5. Loss Function: Cross Entropy

\subsection{Proposed Algorithm}

In proposed algorithm, forward pass and backward propagation algorithm 160 has been implemented. 


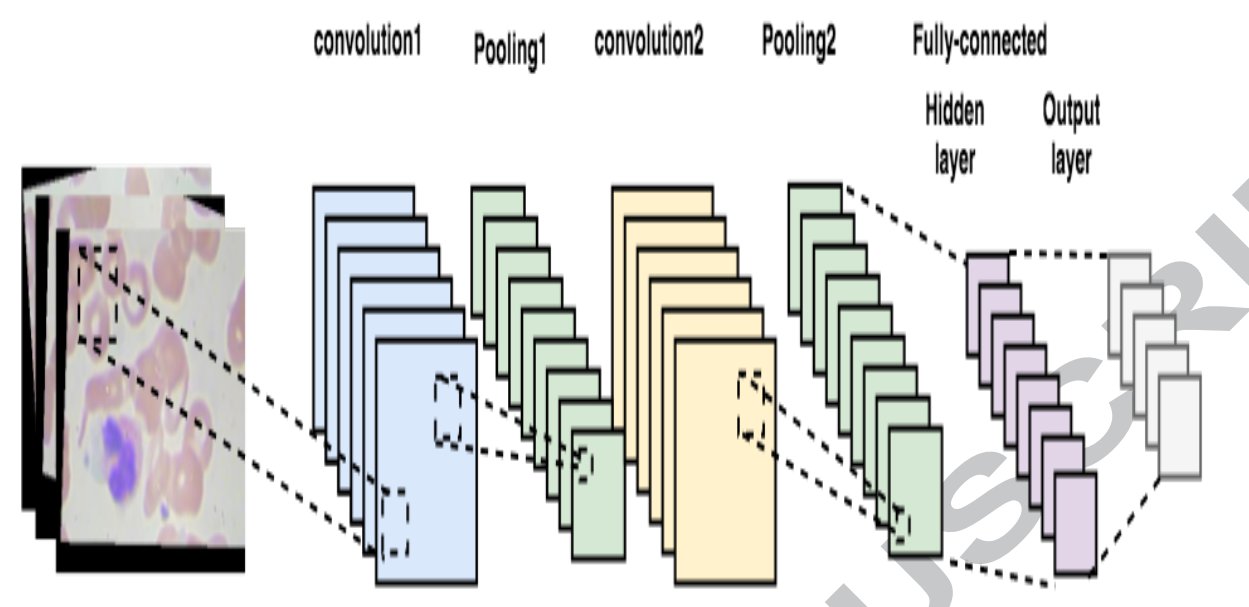

Figure 3: Architecture of the Proposed Model

Forward Pass: Output $\left(P_{x, y}^{l, j}\right)$ of the given neuron of $j^{\text {th }}$ row, $k$ column in the given $l^{\text {th }}$ convolution layer with the $j^{\text {th }}$ feature:

$$
P_{x, y}^{l, j}=\tanh \left(\sum_{r=0}^{j_{h}} \sum_{t=0}^{f-1} \sum_{c=0}^{j_{w}} W_{r, c}^{j, t} P_{x+r, x+c}^{l-1, t}+b i a s^{l, j}\right)
$$

where $f$ is the no. of convolution cores in the pattern of feature. $\left(P_{x, y}^{l, j}\right)$ neuron of the given row $x$ and $y$ column in the $l^{\text {th }}$ subsample layer with the $j^{\text {th }}$ feature:

$$
P_{x, y}^{l, j}=\tanh \left(W^{k} \sum_{r=0}^{S_{h}} \sum_{c=0}^{S_{w}} P_{x_{S_{h}}+r, y_{S_{w}}+c}^{l-1, t}+\text { bias }^{l, j}\right)
$$

so the output of the $k_{t h}$ neuron in the $l_{t h}$ hidden layer $H$ will be:

$$
P_{l, k}=\tanh \left(\sum_{x=0}^{S_{h}} \sum_{j=0}^{S-1} \sum_{y=0}^{S_{w}} W_{x, y}^{k, j} P_{x, y}^{l-1, t}+\text { bias }^{l, k}\right)
$$

where number of pattern feature in simple layer is represented by $s$ here so output of the $i^{\text {th }}$ neuron and $l^{\text {th }}$ output layer $P$

$$
P_{l, i}=\tanh \left(\sum_{k=0}^{H} W_{i, k}^{l} P_{l-1, k}+\text { bias }^{l, i}\right)
$$

Back Propagation: Output deviation of the $j_{t h}$ neuron in the output layer $o$ can be written as $d\left(P_{j}^{o}\right)=y_{j}-t_{j}$ and the input deviation of the $j_{t h}$ neuron 
in the output layer is $d\left(I_{j}^{o}\right)=y_{j}-t_{j} \delta^{\prime}\left(v_{j}\right)=\delta^{\prime}\left(v_{j} d\left(P_{j}^{o}\right)\right.$. Bias and weight variation of the $j_{t h}$ neuron in the output is $\Delta b i a s_{j}^{o}=d\left(I_{j}^{o}\right), \Delta W_{j, x}^{o}=d\left(P_{j}^{o}\right) y_{j, x}$. In the hidden layer $H$, output bias of the $j_{t h}$ neuron is $d\left(P_{j}^{H}\right)=\sum_{i=0}^{i<a} d\left(I_{i}^{o}\right) W_{i, j}$ where $a$ is the number of different pictures. In the hidden layer $H$, input bias of the $j_{t h}$ neuron is $d\left(I_{j}^{H}\right)=\delta^{\prime}\left(v_{j}\right) d\left(P_{j}^{H}\right)$. Bias and weight variation in the column $y$ and row $x$ in the $n^{\text {th }}$ feature pattern, previous layer in the head of $j^{\text {th }}$ neurons in the hidden layer $\mathrm{H}$ is $\Delta \operatorname{bias}_{j}^{H}=d\left(I_{j}^{H}\right), \Delta W_{n, x, y}^{H, j}=d\left(I_{j}^{H}\right) y_{x, y}^{n}$. Output bias of column $y$ and row $x$ in the $n^{\text {th }}$ feature pattern with $S$ sub sample layer is $d\left(P_{x, y}^{S, n}\right)=\sum_{k}^{b} d\left(I_{n, x, y}^{H}\right) W_{n, x, y}^{H, j}$ where $b$ is the number of neurons in the hidden layer $H$. Input bias of column $y$ and row $x$ in the $n^{\text {th }}$ feature pattern with $S$ sub sample layer is $d\left(I_{x, y}^{S, n}\right)=\delta^{\prime}\left(v_{j}\right) d\left(P_{x, y}^{S, n}\right)$. Bias and weight variation in the column $y$ and row $x$ in the $n^{\text {th }}$ feature pattern, $S$ sub sample layer is $\Delta$ bias $^{S, n}=\sum_{y=0}^{f_{w}} \sum_{x=0}^{f_{h}} d\left(P_{x, y}^{S, n}\right), \Delta W(S, n)=\sum_{y=0}^{f_{w}} \sum_{x=0}^{f_{h}} d\left(I_{x / 2, y / 2}^{S, n}\right) P_{x, y}^{C, n}$. Convolution layer is represented by $C$ here. So, Output bias of column $y$ and row $x$ in the $j^{\text {th }}$ feature pattern with $C$ convolution layer can be written as :

$$
d\left(P_{x, y}^{C, j}\right)=d\left(I_{x / 2, y / 2}^{S, j}\right) W_{j}
$$

Input bias of column $y$ and row $x$ in the $j^{\text {th }}$ feature pattern with $C$ convolution layer can be written as:

$$
d\left(I_{x, y}^{C, j}\right)=\delta^{\prime}\left(v_{j}\right) d\left(P_{x, y}^{C, j}\right)
$$

Weight variation of column $c$ and row $r$ in the $n^{t h}$ convolution core in addition with $j^{\text {th }}$ feature pattern the given $l^{\text {th }}$ layer with $C$ convolution layer.

$$
\Delta W_{r, c}^{j, n}=\sum_{y=0}^{f_{w}} \sum_{x=0}^{f_{h}} d\left(I_{x, y}^{C, j}\right) P_{x+r, y+c}^{l-1, n}
$$

So the total bias variation can be written of the convolution core

$$
\Delta \text { bias }^{C, j}=\sum_{y=0}^{f_{w}} \sum_{x=0}^{f_{h}} d\left(I_{x, y}^{C, j}\right)
$$

\subsection{Evaluation Parameters}

There are some parameters often used in machine learning and deep learning in order to check the performance of the proposed model. Accuracy, Precision, 
Recall and F-measure has been used to evaluate the performance of the models and that is mentioned in the given equations. confusion matrix can be seen from the given table

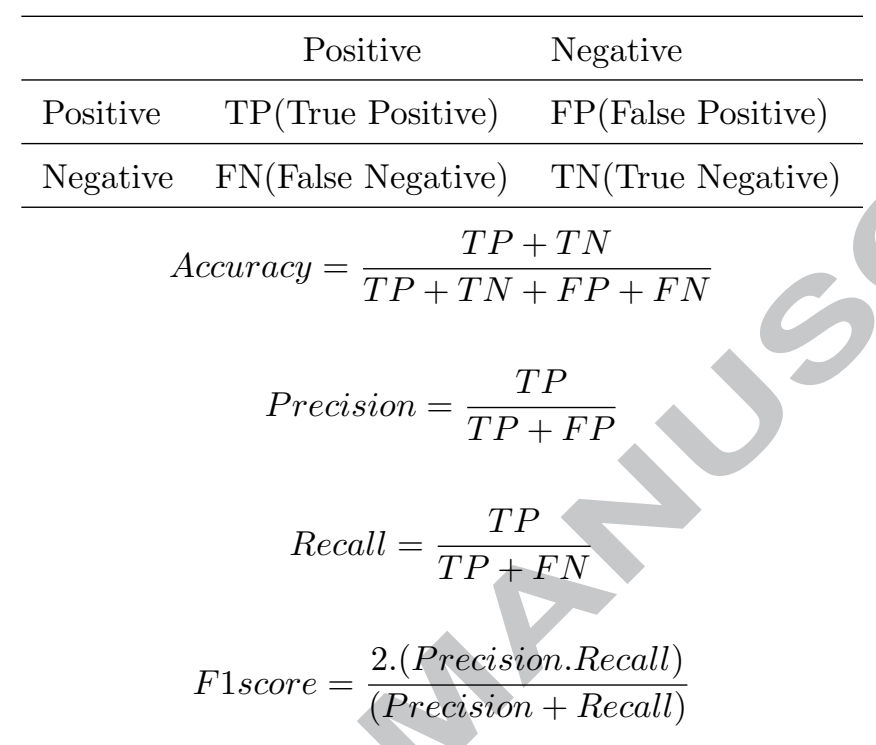

\section{Data Record}

The experiment dataset contains around 13k augmented pictures of blood cells with having the blood cell type labels. There are around 3000 pictures for each blood cell types which are into 5 different categories with the type of cell i.e. eosinphil, basophil,lymphocyte, neutrophil and monocyte. Basophil is not taken into consideration during analysis because there was no image of basophil in the obtained dataset. There is original dataset accompanied with augmented one which has 400 pictures in jpeg file with having two blood cell labels i.e. polynuclear and mononuclear. More details about dataset can be found here https://bigpictureeducation.com/blood-cells-images.

\section{Experiments}

\subsection{Setups}

180

For this analysis, these setups has been utilized in order to achieve the result and can be seen below. 


\begin{tabular}{lc}
\hline Processor & Intel(R) Core(TM)i7-7700K CPU @ 4.20GHz \\
\hline RAM & $16.0 \mathrm{~GB}$ \\
\hline Operating System & Ubuntu 18.04 \\
\hline Graphic Card & Nvidia GeForce GTX 1060 3GB \\
\hline
\end{tabular}

\subsection{Results Analysis and Discussion}

In the first array of experiments, we validate our proposed double convoprecision of neutrophil classifier, recall of the eosinophil classifier, etc. However, comprehensively MaxPooling outperforms AveragePooling shown in table 2. The average performance(precision, recall and F1 score) is graphically demonstrated in Figure 4, 5, 6. Referring to two classes, they are virtually identical. and validation we exhaustively iterate variant number and compute the precision, in Figure 7. The red line indicates the training precision and the blue line is the validation precision. We conclude that 25 is a good option trade-off between 
precision and computing time. Our proposed model outperform the traditional machine learning models which can be very useful in the medical field and this proposed model allow to get rid from the blood diagnosis problems.

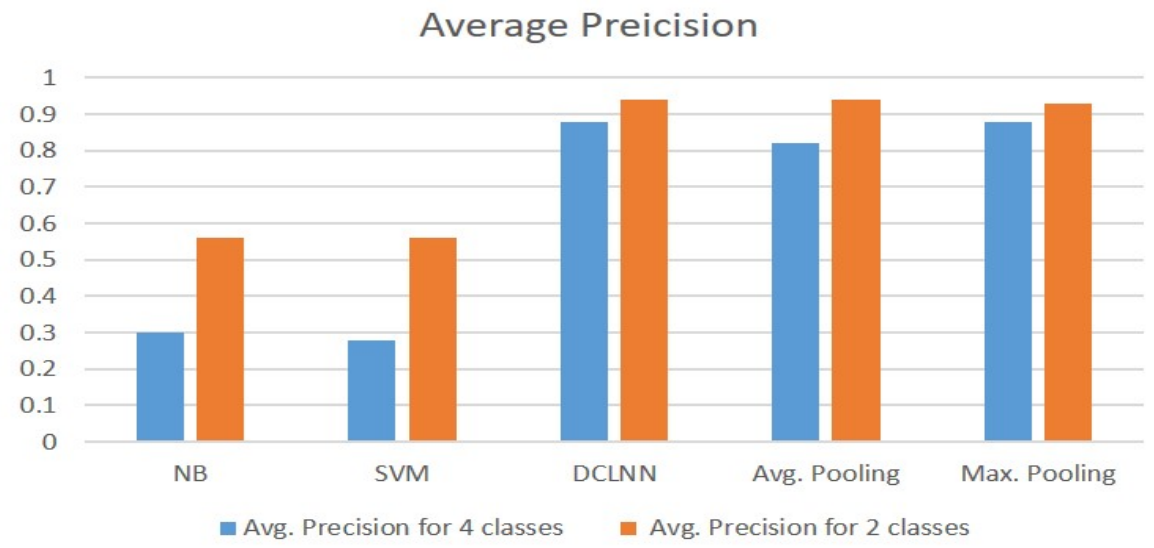

Figure 4: Average Precision comparison among Naive Bayes, Support Vector Machine, Double Convolution Layer Neural Network (DCLNN), Average Pooling and Max Pooling based on two classes and four classes

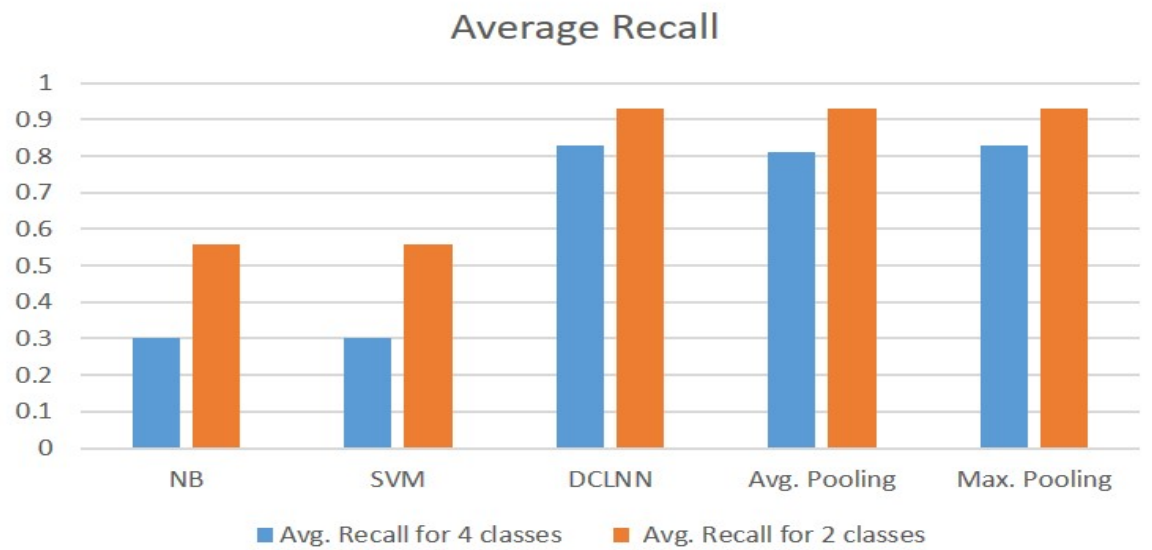

Figure 5: Average Recall comparison among Naive Bayes, Support Vector Machine, Double Convolution Layer Neural Network (DCLNN), Average Pooling and Max Pooling based on two classes and four classes 


\begin{tabular}{|l|l|l|l|l|}
\hline \multicolumn{5}{|c|}{ four classes } \\
\hline Method & Class & Precision & Recall & F1 score \\
\hline NB & NEUTROPHIL & 0.28 & 0.32 & 0.29 \\
& EOSINOPHIL & 0.28 & 0.26 & 0.27 \\
& MONOCYTE & 0.39 & 0.41 & 0.40 \\
& LYMPHOCYTE & 0.25 & 0.22 & 0.23 \\
& Average & 0.30 & 0.30 & 0.30 \\
\hline SVM & NEUTROPHIL & 0.28 & 0.27 & 0.27 \\
& EOSINOPHIL & 0.27 & 0.31 & 0.29 \\
& MONOCYTE & 0.40 & 0.50 & 0.44 \\
& LYMPHOCYTE & 0.17 & 0.11 & 0.13 \\
& Average & 0.28 & 0.30 & 0.28 \\
\hline DCLNN & NEUTROPHIL & $\mathbf{0 . 6}$ & $\mathbf{0 . 9 4}$ & $\mathbf{0 . 7 4}$ \\
& EOSINOPHIL & $\mathbf{0 . 9 5}$ & $\mathbf{0 . 6 1}$ & $\mathbf{0 . 7 4}$ \\
& MONOCYTE & $\mathbf{0 . 9 7}$ & $\mathbf{0 . 7 7}$ & $\mathbf{0 . 8 6}$ \\
\hline SVM & LYMPHOCYTE & $\mathbf{1}$ & $\mathbf{1}$ & $\mathbf{1}$ \\
\hline \multirow{2}{*}{ NB } & Average & 0.88 & 0.83 & 0.83 \\
\hline & Aononuclear & 0.56 & 0.52 & 0.54 \\
\hline & Aolynuclear & 0.55 & 0.59 & 0.57 \\
& Average & 0.56 & 0.56 & 0.56 \\
\hline & Mononuclear & 0.56 & 0.51 & 0.54 \\
& Polynuclear & 0.56 & 0.61 & 0.58 \\
& Average & 0.56 & 0.56 & 0.56 \\
\hline
\end{tabular}

Table 1: Compare Double Convolution Layer Neural Network (DCLNN) with NB and SVM in terms of two classes and four classes 


\begin{tabular}{|l|l|l|l|l|}
\hline \multicolumn{5}{|c|}{ MaxPooling VS AveragePooling (four classes) } \\
\hline Method & Class & Precision & Recall & F1 score \\
\hline AveragePooling NEUTROPHIL & $\mathbf{0 . 6 4}$ & 0.81 & 0.71 \\
& EOSINOPHIL & 0.81 & $\mathbf{0 . 6 3}$ & 0.71 \\
& MONOCYTE & 0.94 & $\mathbf{0 . 8}$ & $\mathbf{0 . 8 6}$ \\
& LYMPHOCYTE & 0.91 & $\mathbf{1}$ & 0.95 \\
& Average & 0.82 & 0.81 & 0.81 \\
\hline MaxPooling & NEUTROPHIL & 0.60 & $\mathbf{0 . 9 4}$ & $\mathbf{0 . 7 4}$ \\
& EOSINOPHIL & $\mathbf{0 . 9 5}$ & 0.61 & $\mathbf{0 . 7 4}$ \\
& MONOCYTE & $\mathbf{0 . 9 7}$ & 0.77 & $\mathbf{0 . 8 6}$ \\
& LYMPHOCYTE & $\mathbf{1}$ & $\mathbf{1}$ & $\mathbf{1}$ \\
& Average & 0.88 & 0.83 & 0.83 \\
\hline \multirow{5}{*}{ AveragePooling } & MaxPooling VS AveragePooling (two classes) \\
& Mononuclear & $\mathbf{0 . 9 9}$ & 0.87 & $\mathbf{0 . 9 3}$ \\
& Polynuclear & $\mathbf{0 . 8 9}$ & $\mathbf{0 . 9 9}$ & $\mathbf{0 . 9 4}$ \\
& Average & 0.94 & 0.93 & 0.93 \\
\hline MaxPooling & Mononuclear & 0.98 & $\mathbf{0 . 8 8}$ & 0.92 \\
& Polynuclear & $\mathbf{0 . 8 9}$ & 0.98 & 0.93 \\
& Average & 0.93 & 0.93 & 0.93 \\
\hline
\end{tabular}

Table 2: Compare MaxPooling and AveragePooling in terms of 2 classes and four classes

\section{Conclusion and Future Works}

From the obtained experiments, Our proposed models suggest that implementation of deep learning enhance the classification task as compared to stateof-art models. SVM and Naive Bayes has been utilized as a baseline to compare with the proposed CNN based model (DCLNN) and it compete in all aspects with the baseline approaches. Our proposed model can automatically classify the blood cell images into subtypes of the cells with high accuracy, precision and other evaluation parameters. This proposed model can be very beneficial for blood diagnosis in the medical field that can save a lot of time. We believe that 


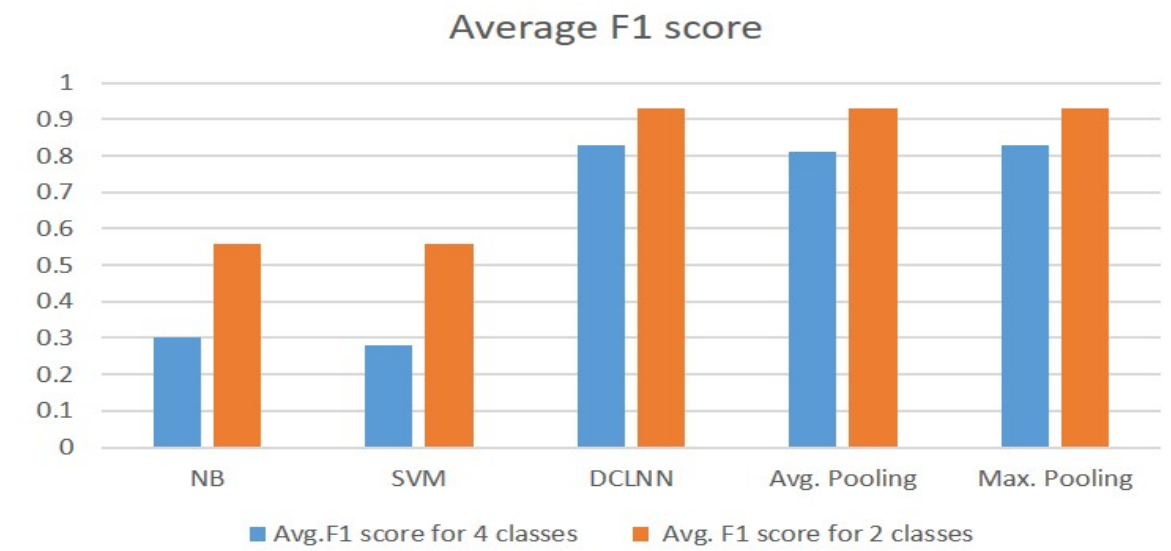

Figure 6: Average F1 score comparison among Naive Bayes, Support Vector Machine, Double Convolution Layer Neural Network (DCLNN), Average Pooling and Max Pooling based on two classes and four classes

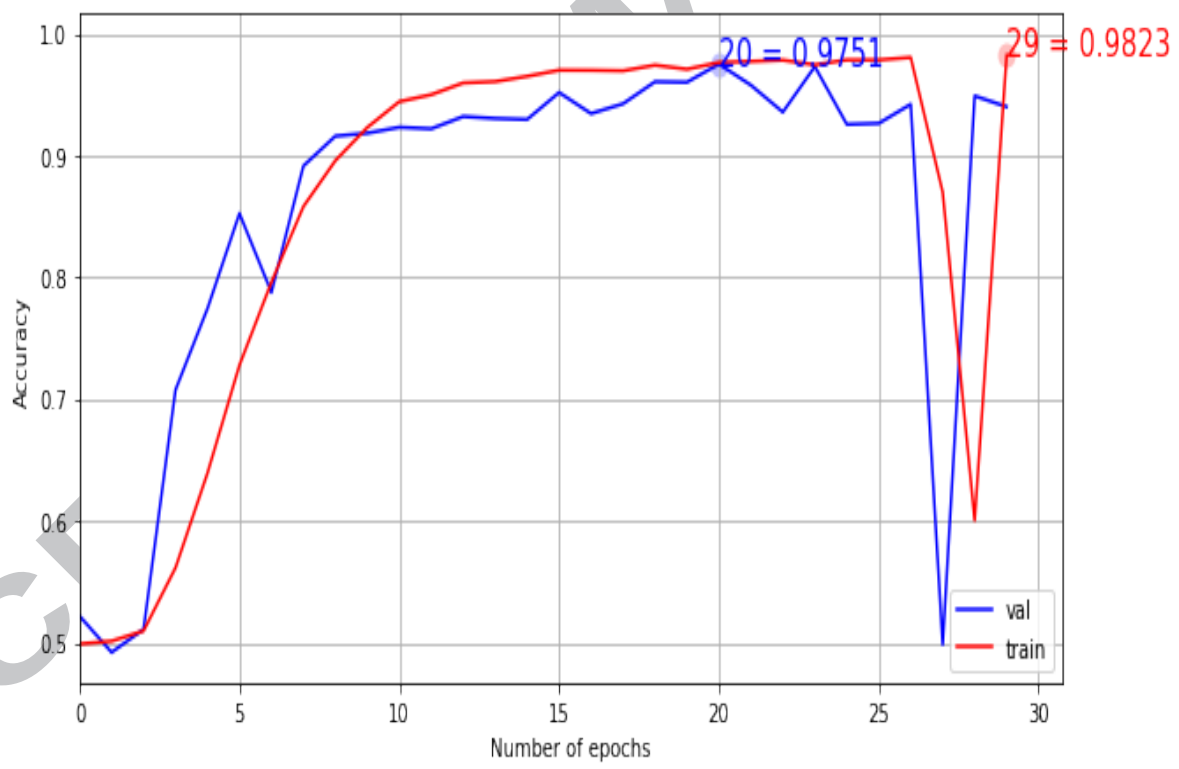

Figure 7: Number of epochs with accuracy

there is always room for improvement in every field so as well in this field also. Researchers may implement this work on large dataset that may outperform the 

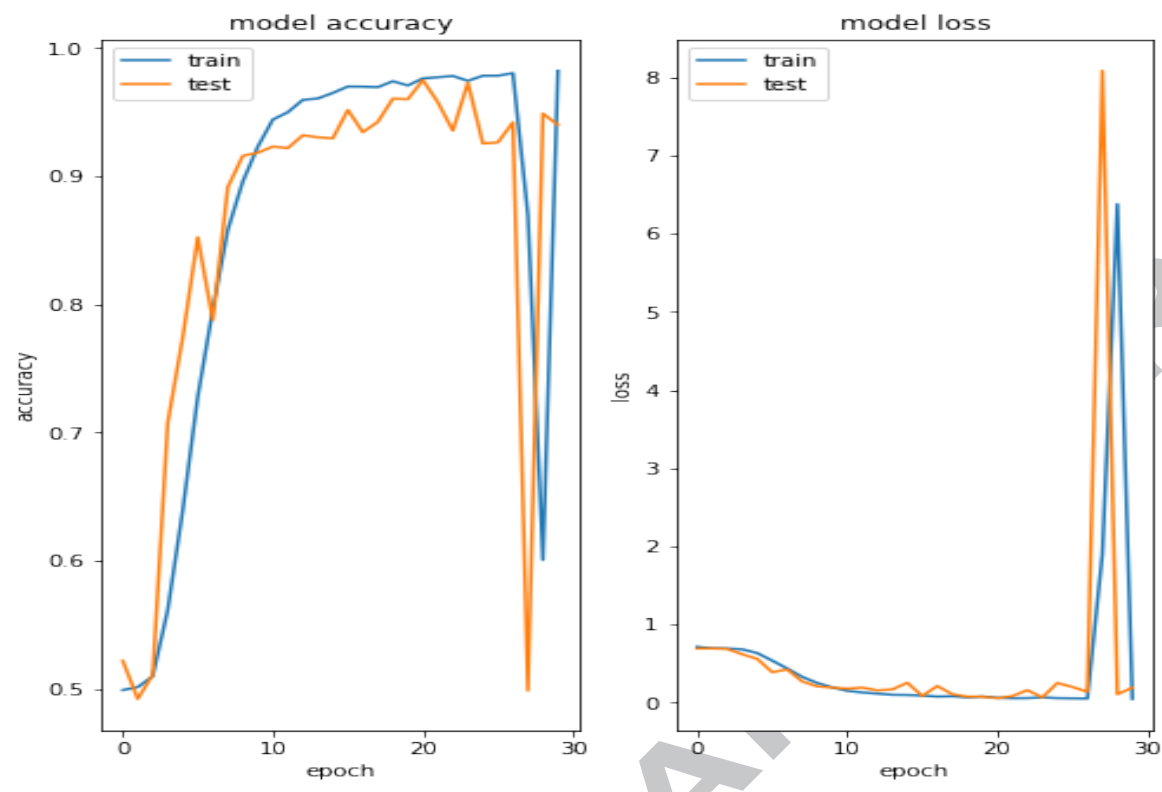

Figure 8: Model accuracy and loss

current results.

\section{References}

225 [1] C. A. Doan, The white blood cells in health and disease, Bulletin of the New York Academy of Medicine 30 (6) (1954) 415.

[2] D. M. U. Sabino, L. da Fontoura Costa, E. G. Rizzatti, M. A. Zago, A texture approach to leukocyte recognition, Real-Time Imaging 10 (4) (2004) 205-216.

[3] M.-C. Su, C.-Y. Cheng, P.-C. Wang, A neural-network-based approach to white blood cell classification, The scientific world journal 2014.

[4] H. Sheikh, B. Zhu, E. Micheli-Tzanakou, Blood cell identification using neural networks, in: Bioengineering Conference, 1996., Proceedings of the 1996 IEEE Twenty-Second Annual Northeast, IEEE, 1996, pp. 119-120. 
[10] S. H. Rezatofighi, H. Soltanian-Zadeh, Automatic recognition of five types of white blood cells in peripheral blood, Computerized Medical Imaging and Graphics 35 (4) (2011) 333-343.

[11] I. Cseke, A fast segmentation scheme for white blood cell images, in: Pattern Recognition, 1992. Vol. III. Conference C: Image, Speech and Signal Analysis, Proceedings., 11th IAPR International Conference on, IEEE, 1992, pp. 530-533. 
[12] J.-M. Chassery, C. Garbay, An iterative segmentation method based on a contextual color and shape criterion, IEEE Transactions on Pattern Analysis and Machine Intelligence (6) (1984) 794-800.

[13] K. Jiang, Q.-M. Liao, S.-Y. Dai, A novel white blood cell segmentation scheme using scale-space filtering and watershed clustering, in: Machine Learning and Cybernetics, 2003 International Conference on, Vol. 5, IEEE, 2003, pp. 2820-2825.

[14] N. H. Harun, M. Y. Mashor, R. Hassan, Segmentation technique for acute leukemia blood cells images using saturation component and moving $\mathrm{k}-$ mean clustering procedures.

[15] J. Wu, P. Zeng, Y. Zhou, C. Olivier, A novel color image segmentation method and its application to white blood cell image analysis, in: Signal Processing, 2006 8th International Conference on, Vol. 2, IEEE, 2006.

[16] A. A. Salihah, M. Mashor, N. H. Harun, A. A. Abdullah, H. Rosline, Improving colour image segmentation on acute myelogenous leukaemia images using contrast enhancement techniques, in: Biomedical Engineering and Sciences (IECBES), 2010 IEEE EMBS Conference on, IEEE, 2010, pp. $246-251$.

[17] R. Adollah, M. Mashor, N. M. Nasir, H. Rosline, H. Mahsin, H. Adilah, Blood cell image segmentation: a review, in: 4th Kuala Lumpur International Conference on Biomedical Engineering 2008, Springer, 2008, pp. $141-144$.

[18] L. Putzu, C. Di Ruberto, White blood cells identification and classification from leukemic blood image, in: International Work-Conference on Bioinformatics and Biomedical Engineering, Copicentro Editorial, 2013, pp. 99-106. 